\title{
A key role of gut microbiota-vagus nerve axis and gut microbiota-spleen axis in sleep deprivation- mediated aggravation of systemic inflammation after LPS administration
}

\author{
Yujing Zhang \\ Wuhan Union Hospital \\ Bing Xie \\ Wuhan Union Hospital \\ Zheng LV \\ Wuhan Union Hospital \\ Hong Qi \\ Wuhan Union Hospital \\ Jiancheng Zhang \\ Wuhan Union Hospital
}

Shiying Yuan ( $\nabla$ yuan_shiying@163.com )

Union Hospital, Tongji Medical College, Huazhong University of Science and Technology

\section{Research}

Keywords: Gut microbiota; Lipopolysaccharide; Sleep deprivation; Spleen; Subdiaphragmatic vagus nerve.

Posted Date: August 5th, 2020

DOI: https://doi.org/10.21203/rs.3.rs-52216/v1

License: (c) This work is licensed under a Creative Commons Attribution 4.0 International License.

Read Full License 


\section{Abstract}

Background: Sleep deprivation (SD) is shown to be correlated with exacerbated systemic inflammation after sepsis. However, the underlying mechanisms remain unclear.

Methods: In this study, mice were intraperitoneally injected with lipopolysaccharide (LPS) followed by 3 consecutive days of SD. The subdiaphragmatic vagotomy (SDV) was performed 2 weeks before LPS injection. The pseudo germ-free mouse model was created by administering antibiotics for 14 consecutive days, and then fecal microbiota transplant (FMT) was performed by gavaging supernatant from fecal suspension of septic mice with or without SD into pseudo germ-free mice with or without SDV or splenectomy. Splenectomy was performed 14 days prior to antibiotics administration.

Results: We found that SD after LPS administration increased the plasma levels of interleukin-6 (IL-6) and tumor necrosis factor-a (TNF-a), reduced IL-10 plasma leve, increased spleen weight, and promoted pathological injury and IL-6 expression in lung, liver and kidney. Post-septic sleep deprivation had no effects on the diversity of gut microbiota. However, the relative abundance of Proteobacteria and its subgroups were increased in septic mice received SD. Pseudo germ-free mice transplanted with fecal bacteria from septic mice subjected to SD developed splenomegaly, systemic inflammation, organ inflammation and damage as their donors do. Intriguingly, SDV abolished the aggravated effects of SD on splenomegaly and inflammatory organ injury in septic mice received SD or in pseudo germ-free mice transplanted with fecal bacteria from septic mice subjected to SD. Furthermore, Splenectomy also abrogated the increase in IL-6 and TNF-a plasma levels and the decrease in IL-10 plasma level in pseudo germ-free mice transplanted with fecal bacteria from septic mice subjected to SD.

Conclusions: Taken together, our results suggest that gut microbiota-vagus nerve axis and gut microbiota-spleen axis could play key roles in modulating systemic inflammation induced by SD after LPS administration.

\section{Introduction}

Sepsis, a systemic inflammatory response to an infectious insult, is the leading cause of death in critically ill patients [1]. Early mortality in sepsis are mainly due to excessive inflammatory response, which is characterized by a strong activation of the innate immune system [2]. Sleep and the circadian system could exert a strong regulatory influence on immune function. It has been reported that sleep and circadian rhythm are severely abnormal in critically ill patients [3-6]. Changes in circadian rhythm after sepsis can aggravate the disease and increase mortality [3]. Evidence has shown that prolonged sleep deprivation (SD) can invoke a persistent production of pro-inflammatory cytokines such as interleukin (IL)-1,IL-6 and tumor necrosis factor-a (TNF-a), and produce a chronic low-grade inflammation, which are potentially expand the pathological sequelae of sepsis [7-10]. However, the underlying roles and mechanisms of SD in systemic inflammation after lipopolysaccharide administration remain unclear. 
Sleep loss could induce subtle effects on human microbiota. In a randomized within-subject crossover study, microbiota composition analysis revealed that compared with two days of normal sleep, individuals experiencing two days of partial SD exhibited an obvious fecal microbial disorder [11]. Chronic sleep fragmentation for 4 weeks could also alter the gut microbiota composition in mice [12]. The abnormal gut microbiota composition could cause colonic epithelium barrier disruption and lead to systemic and visceral white adipose tissue inflammation [12], suggesting that gut microbiota has an important role in regulating immuno-inflammatory responses. Studies have demonstrated that gut microbiota is responsible for inducing $80 \%$ immune responses [13]. However, the roles and mechanisms of gut microbiome in systemic inflammation exacerbated by SD after lipopolysaccharide (LPS) administration are unclear.

Previous studies have shown that stimulation of cervical vagus nerve can decrease the production of proinflammatory cytokines in experimental models of systemic inflammation, including lethal endotoxemia, hemorrhagic shock, and ischemia-reperfusion injury [14-18]. However, we previously found that subdiaphragmatic vagotomy (SDV) performed 14 days prior to LPS injection could attenuate intraperitoneally injection with $0.5 \mathrm{mg} / \mathrm{kg}$ LPS-mediated intestinal flora disorder, and increase in spleen weight and plasma levels of IL-6 and TNF-a in mice [19], indicating that different branches of the vagus nerve could play different roles under pathological conditions. We speculated that the dorminated specific microorganisms in the intestinal flora after LPS treatment might lead to systemic inflammation through the subdiaphragmatic vagus nerve. The roles and mechanisms of the subdiaphragmatic vagus nerve in systemic inflammation in mice treated with LPS followed by 3 consecutive days of SD remain unclear.

Spleen, as the largest secondary lymphoid organ in the body, hosts a wide range of immunological and inflammatory regulatory effects [20]. It is shown that neonatal gut microbiota exert critical roles in spleen development [21]. Spleen is essential for gut microbiota disorder-induced postoperative immunosuppression after sleep restriction, which is mediated by subdiaphragmatic vagus nerve [22]. In our previous study, we found that removal of the spleen 14 days prior to repeated short-term sleep restriction (CRSR) abrogated the aggravated effects of CRSR on LPS ( $5 \mathrm{mg} / \mathrm{kg})$-induced increase in the plasma levels of IL-6, TNF-a, interferon gamma (IFN- $y$ ) and IL-17A [23], indicating an essential role of spleen in modulating systemic inflammation after sleep disorder followed by LPS administration. However, the roles and mechanisms of spleen on the aggravated effects of altered gut microbiota after SD on systemic inflammation after LPS administration are not clear.

Here, we demonstrated that 3 consecutive days of SD after LPS administration could aggravate systemic inflammation and inflammatory organ injury. SDV could alleviate post-septic SD-mediated aggravation of systemic inflammation and prevent changes in gut microbiota composition induced by SD after LPS administration. Our findings demonstrated that gut microbiota-subdiaphragmatic vagus nerve axis could play important roles in the aggravated effects of SD on systemic inflammation after sepsis.

\section{Methods}




\section{Animals}

Male C57BL/6J mice (8-10 weeks old, weighing 20-25 g) were obtained from Vital River Laboratory Animal Technology Co Ltd, Beijing, China. Mice were housed and maintained on a 12:12 light-dark cycle with ad libitum access to food and water under specific pathogen free conditions. All procedures were performed in accordance with the National Institute of Health $(\mathrm{NIH})$ Guide for the Care and Use of Laboratory Animals (publications no.80-23) revised 1996. All animal protocols were approved by the committee of experimental animals of Tongji Medical College (Wuhan, China).

\section{Experimental grouping and treatment}

The experiment was performed in three parts. In part A, sleep-deprived or non-sleep-deprived groups with or without SDV 14 days prior to LPS administration. Mice were intraperitoneally administrated with LPS (5 mg/kg; L-4130, serotype 0111:B4; Sigma-Aldrich, St Louis, MO) or 0.9\% saline (5 ml/kg). SDV or sham operation was performed 14 days prior to LPS administration. SD was performed immediately after LPS administration for 3 consecutive days. Mice were euthanized to collect plasma, spleen, lung, liver, kidney and fecal samples on day 4 after LPS administration.

In part B, control pseudo germ-free mice or pseudo germ-free mice with SDV or sham-operation were received fecal microbiota transplantation (FMT) from LPS-treated mice with or without SD. Pseudo germfree mice underwent SDV or sham operation 14 days prior to antibiotics administration. Mice were euthanized and plasma, lung, liver and kidney were collected after the commencement of FMT.

In part C, pseudo germ-free mice with splenectomy or sham-operation were received FMT from LPStreated mice with or without SD. Mice were euthanized and plasma was collected after the commencement of FMT. Splenectomy was performed 14 days prior to antibiotics administration.

\section{Sleep deprivation model}

Sleep deprivation was performed using a modified multiple platform method $[24,25]$. Mice were placed in a ventilated transparent cage $(50 \times 30 \times 17 \mathrm{~cm} ; 3$ mice/cage) containing 9 circular platform $(3.5 \mathrm{~cm}$ in diameter) surrounded by water up to $1 \mathrm{~cm}$ beneath the upper surface of the platforms enabling the mice to move between the platforms. When the mice had reached the rapid eye movement stage of sleep, muscle atonia could cause the mouse to fall into the water. The water in the cage was replaced with clean water every day. Food and water were available ad libitum throughout the sleep-deprivation period. The non-sleep-deprived mice were maintained under normal feeding conditions after administering LPS.

\section{Total SDV}

Bilateral SDV was performed under anesthesia with $5 \%$ isoflurane..Upper abdominal wall was opened through a $1 \mathrm{~cm}$ right transverse abdominal incision. Esophagus was exposed by carefully keeping costal arc, liver, and stomach out of sight. The ventral and dorsal branch of the vagus nerve was exposed along the subdiaphragmatic esophagus under a surgical microscope (BX51, Olympus, Japan). Each vagal 
branch was ligated twice with a surgical thread at an interval of $1 \mathrm{~cm}$, and then dissected between the ligatures. After fluid resuscitation with $0.5 \mathrm{~mL}$ sterile saline solution, the abdominal wall was closed. For sham surgery, the vagus nerve were exposed but not cut.

\section{Splenectomy}

Total splenectomy was performed under the isoflurane anesthesia. Splenectomy was performed by making a 2-cm subcostal incision on the left dorsolateral side of the abdomen, and the spleen was carefully exteriorized through the incision. The afferent and efferent vessels near to the spleen were ligated using 6-0 silk suture and the spleen was removed. The abdominal wall was closed with 4-0 silk suture and the skin was sutured using 3-0 silk suture. Sham-splenectomized mice underwent laparotomy without removal of the spleen.

\section{Pseudo germ-free mice modeling}

Based on a previous study [26], broad-spectrum antibiotics (ampicillin $1 \mathrm{~g} / \mathrm{L}$, neomycin sulfate $1 \mathrm{~g} / \mathrm{L}$, and metronidazole $1 \mathrm{~g} / \mathrm{L}$; Sigma-Aldrich Co. Ltd, USA) dissolved in drinking water were given ad libitum to mice for 14 consecutive days. The drinking solution was renewed every 2 days.

\section{FMT}

The fecal donor mice received continuous 3 days of SD or non-sleep-deprivation after LPS administration. On day 4, mice were placed in a clean cage with sterilized filter paper on the bottom. Stool samples from the experimental mice were collected immediately after defecation in a sterile micro-tube and immediately stored in a $-80^{\circ} \mathrm{C}$ freezer until analysis. $1 \mathrm{~g}$ of fecal sample from donor mice was diluted in $10 \mathrm{~mL}$ of phosphate-buffered saline (PBS). The feces were then suspended and $200 \mu \mathrm{L}$ of the suspension was given to each mouse recipient by gavage.

\section{Enzyme-linked immunosorbent assay (ELISA)}

Blood was collected via cardiac puncture, placed into tubes containing ethylenediaminetetraacetic acid (EDTA), and immediately centrifuged at $3,000^{\prime} \mathrm{g}$ for $5 \mathrm{~min}$ at $4^{\circ} \mathrm{C}$, to obtain plasma, and then stored at $80^{\circ} \mathrm{C}$ until bioanalysis. The commercial kits were used for the quantitative assay of IL- 6 (Cat Number: 887064, Invitrogen, Camarillo, CA, USA), IL-10 (Cat Number: EMC005.96, NeoBioscience, China) and TNF-a (Cat Number: 88-7324, Invitrogen, Camarillo, CA, USA) according to the manufacturer's instructions.

\section{Stool sample collection and microbial sequencing}

Fecal samples were collected and placed in $1.5 \mathrm{ml}$ tubes, snap-frozen on dry ice, and stored at $-80^{\circ} \mathrm{C}$. The 16S rRNA analysis of the fecal samples was performed by GENEWIZ Biotech Co., Ltd. (Suzhou, China). Briefly, total genome DNA from samples was extracted using Soil DNA Kit according to manufacturer's protocols. DNA samples were PCR-amplified in in $25 \mu \mathrm{L}$ triplicate reactions with bacterial 16S rRNA gene (V3-V5 region)-specific forward primers containing the sequence "ACTCCTACGGGAGGCAGC" and 
reverse primers containing the sequence "GGACTACHVGGGTWTCTAAT". The amplified DNA libraries were validated and quantified for sequencing on an Illumina MiSeq instrument (Illumina, San Diego, CA, USA). Through quality filtering out, the effective sequences were grouped into operational taxonomic units (OTUs). a-diversity and $\beta$-diversity were performed by the MOTHUR program.

\section{Periodic acid-schiff (PAS) staining and hematoxylin-eosin (HE) staining}

Spleen, lung, liver and kidney tissues were collected and immediately fixed in $4 \%$ paraformaldehyde (pH 7.4) at $4^{\circ} \mathrm{C}$ for 48 hours and then embedded in paraffin for sectioning at $5 \mu \mathrm{m}$. Paraffin-embedded kidney tissue sections were stained with PAS stain according to a standard protocol. Paraffin-embedded Lung, liver and spleen tissue sections were stained with HE. Stained sections were photographed under light microscopy (BX51, Olympus, Japan).

\section{Western blotting}

The lung, liver and kidney samples were lysed in RIPA lysis buffer containing protease inhibitors (KeyGen Biotech, Nanjing, China). An equal amount of protein was resolved on a 10\% SDS-PAGE gel (Beyotime Institute of Biotechnology, Shanghai, China) and blotted to a PVDF membrane (Millipore; Merck KGaA) using an electrophoresis apparatus (Bio-Rad Laboratories, Inc., Hercules, CA, United States). Membranes were blocked with $5 \%$ skim milk diluted in Tris-buffered saline containing $0.1 \%$ tween-20 (TBST) at room temperature for 1 hour. Subsequently, the membranes were incubated with primary antibodies against IL6 (1:500; ABclonal Biotechnology, Co., Ltd., Wuhan, China) and $\beta$-actin (1:2,000; Proteintech group, Wuhan, China) overnight at $4^{\circ} \mathrm{C}$. After primary antibody incubation, membranes were washed with TBST and incubated with an appropriate peroxidase-conjugated secondary antibody (1:3000; Proteintech group, Wuhan, China) for 2 hours at room temperature. Following three washes with TBST, chemiluminescent signals were visualized using electrochemiluminescence western blotting detection reagents (Millipore; Merck KGaA) and bands were captured using an UVP gel documentation system (UVP, LLC, Phoenix, AZ, USA). Band intensity was quantified using Image $J$ software (version 1.41; National Institutes of Health, Bethesda, MD, USA).

\section{Quantitative Real-Time PCR (RT-qPCR) measurements}

Lung, liver and kidney samples were homogenized before total RNA isolation using TRIzol reagent (Invitrogen; Thermo Fisher Scientific, Inc., Waltham, MA, USA) according to the manufacturer's protocol. cDNA was obtained using Taqman reverse transcriptase (Applied Biosystems; Thermo Fisher Scientific, Inc.). $\beta$-actin cDNA was amplified using Power SYBR Green (Applied Biosystems; Thermo Fisher Scientific, Inc.). Two step qPCR was performed $\left(95^{\circ} \mathrm{C}\right.$ for $15 \mathrm{~s}, 60^{\circ} \mathrm{C}$ for $60 \mathrm{~s}$ for 40 cycles) with specific primers for IL-6 (forward, 5'-GAGGATACCACTCCCAACAGACC-3'; reverse: 5'-GAGGGATATCTATCAGG GTCTTCAT-3') and $\beta$-actin (forward, 5'-AAGGCCAACCGTGAAAAGAT-3' and reverse, $5^{\prime}$ -

GTGGTACGACCAGAGGCATAC-3'). The relative quantitation value is expressed as $2^{-\Delta \Delta C q}$, where $\triangle C q$ is the difference between the mean $\triangle \mathrm{Cq}$ value of duplicate measurements of the sample and $\beta$-actin control. 


\section{Statistical analysis}

Data were expressed as the mean \pm standard error of mean (SEM). Multiple comparisons were performed by one-way analysis of variance (ANOVA) followed by post-hoc Tukey's multiple comparison tests. Two groups were compared by unpaired student $t$ test. Correlation was analyzed by Pearson correlation. $P<$ 0.05 was considered to be statistically significant. Statistical analyses were performed using Prism 8 software (GraphPad Software).

\section{Results}

\section{Effects of SDV on systemic inflammation in septic mice with SD}

We firstly examined the changes of plasma IL-6, TNF- $\alpha$ and IL-10 levels and spleen weight in sleepdeprived mice after sepsis (Fig. 1a). We found that SD after sepsis significantly increased the plasma levels of IL- 6 and TNF-a $(P<0.01$ and $P<0.05$, respectively $)$ and decreased the plasma IL-10 level $(P<$ 0.05 ), which was abrogated by SDV 14 days prior to LPS administration (Fig. $1 \mathrm{~b}-\mathrm{d}$; all $P<0.05$ ).

Consistent with the changes in plasma IL-6 and TNF-a levels, the spleen weight and spleen/body weight ratio were also higher in sleep-deprived septic mice, compared to that in non-sleep-deprived septic mice (Fig. 1e; both $P<0.01$ ), which was also abrogated by SDV 14 days prior to LPS administration (Fig. 1e; $P$ $<0.001$ and $P<0.01$, respectively). HE staining results showed that SDV attenuated the detrimental effects of SD on the loss of spleen nodules and the destruction of spleen structure after sepsis (Fig. 1f). Interestingly, we found that a positive correlation between spleen weight and plasma levels of IL-6 ( $r=$ $0.627, P=0.005$; Fig. $1 \mathrm{~g})$ or TNF-a $(r=0.604, P=0.008$; Fig. $1 \mathrm{~h})$ among the four groups. Collectively, our data indicated that SDV exerted essential roles in post-septic SD-mediated aggravation of systemic inflammation.

\section{Effects of SDV on lung, liver and kidney injury in septic mice with SD}

We then examined the effects of SDV on lung, liver and kidney injury in sleep-deprived septic mice. HE staining of the lung showed that alveolar and interstitial disorder were obvious in septic mice with SD, compared to that in septic mice without SD, which was attenuated by SDV 14 days prior to LPS administration (Fig. 2a). HE staining of the liver showed that hepatic ballooning and hepatic sinus vascularization were observed in sham-operated septic mice with SD, but not in the other two groups (Fig. 2a). Furthermore, kidney PAS staining also showed the same protective effects of SDV on kidney histology in septic mice with SD (Fig. 2d).

Furthermore, RT-PCR and western blotting results showed that the mRNA and protein levels of IL- 6 in the lung (Fig. 2b; both $P<0.01$ ), liver (Fig. 2c; $P<0.01$ and $P<0.05$, respectively) and kidney (Fig. 2e; both $P<$ 0.01 ) were significantly increased after SD in sham-operated septic mice. SDV abrogated the promoting effects of SD on the increase in the mRNA and protein levels of IL-6 in the lung, liver and kidney of septic mice (Fig. 2b, c, e; all $P<0.05$ ). 


\section{Effects of SDV on fecal microbiota diversity and composition in septic mice with SD}

In light of plenty of evidence demonstrating the crucial role of gut microbiota in sepsis and sleep disorder, we sought to determine whether SDV could affect the gut microbiota diversity after septic SD. a-diversity, as the richness of gut microbiota, can be evaluated by Simpson index, Chao 1 index and Shannon index [27]. No significant differences in the Simpson index, Chao 1 index and Shannon index were observed among the four groups (Fig. 3a-d). There were also no significant differences in 3D-Principal Coordinate Analysis (3D-PCoA) and Principal Component Analysis (PCA) among the four groups (Fig. 3e, f).

However, after analyzing the composition of the gut microbiota, we found that a total of 16 bacteria in the fecal samples were significantly altered among the four groups. At the levels of phylum, class and order, post-septic SD significantly increased the relative abundance of Proteobacteria, Gammaproteobacteria and Enterobacteriales, which was abrogated by SDV (Fig. 4; all $P<0.05$ ). The relative abundance of Firmicutes, Clostridia and Clostridiales in post-septic sleep deprived mice with SDV was significantly increased compared to that in post-septic sleep deprived mice with sham operation (Fig. 4; all $P<0.05$ ).

At the levels of genus and species, post-septic SD significantly increased the relative abundance of Escherichia-Shigella, g_Escherichia Shigela_Unclassified and Bacteroides_dorei, which was abrogated by SDV (Fig. 5a, b, g, h; all $P<0.01$ ). SD significantly decreased the relative abundance of Streptococus, $f_{\_}$Muribaculaceae_Unclassified and g_uncultured_bacterium_Unclassified in sham-operated septic mice (Fig. 5c, d, k; $P<0.05, P<0.01$ and $P<0.05$, respectively). The relative abundance of Streptococus was significantly increased (Fig. 5c; $P<0.05$ ), whereas the relative abundance of Parasutterella and g_Parasuterella_Unclasified was significantly decreased in post-septic sleep-deprived mice with SDV (Fig. $5 e$, j; both $P<0.05)$, compared to that in post-septic sleep-deprived mice with sham operation.

\section{Role of gut microbiota-vagus nerve axis in systemic inflammation}

In view of the striking changes in gut microbiota induced by post-septic SD with or without SDV, we then explored whether gut microbiota plays a role in systemic inflammation through SDV. We created pseudo germ-free mouse model by administering antibiotics at large doses for 14 consecutive days, and then FMT was performed by gavaging supernatant from fecal suspension of septic mice with or without SD into pseudo germ-free mice with or without SDV. We found that the spleen weight (Fig. 6b; $P<0.01$ ), spleen/body weight ratio (Fig. 6 c; $P<0.001$ ), plasma levels of IL-6 and TNF-a (Fig. 6 d, e; both $P<0.001$ ) and mRNA expression of IL-6 in lung, liver and kidney (Fig. 6g-i; $P<0.001, P<0.01$ and $P<0.05$, respectively) were significantly increased, whereas the plasma level of IL-10 (Fig. 6f; $P<0.01$ ) was significantly decreased in pseudo germ-free mice transplanted with fecal suspension from septic mice with SD compared with that in pseudo germ-free mice transplanted with fecal suspension from septic mice without SD, which was abrogated by SDV 14 days prior to antibiotics administration. Our results indicated that gut microbiota played an important role in the aggravative effects of post-septic SD on systemic inflammation through SDV.

\section{Role of gut microbiota-spleen axis in systemic inflammation}


In pseudo germ-free mice underwent sham-operation, FMT with fecal suspension from septic mice with SD significantly increased the plasma levels of IL-6 $(P<0.01)$ and TNF-a $(P<0.05)$ and decreased the plasma IL-10 level $(P<0.05)$ compared with that from septic mice without SD, which was abrogated by splenectomy 14 days prior to antibiotics administration (Fig. 6j-m).

\section{Discussion}

In this study, we demonstrated an essential role of gut microbiota-subdiaphragmatic vagus nerve axis in post-septic SD-mediated aggravation of systemic inflammation and organ injury after LPS-induced sepsis. Three consecutive days of SD after LPS administration increased the levels of proinflammatory cytokines (IL-6 and TNF-a) in the plasma and organs (lung, liver and kidney), which could be abrogated by SDV 14 days prior to LPS administration. A number of studies have reported associations between disrupted sleep/SD and inflammatory responses [28-31]. Chronic circadian misalignment or prolonged sleep restriction could increase the plasma expression of IL-6, TNF-a or C-reactive protein [32, 33]. Acute sleep disturbance (sleep restriction or SD) was associated with increased systemic inflammation in adult humans or mice $[34,35]$. One night of partial SD could increase the production of IL-6 and TNF-a from monocytes that persisted after a night of recovery sleep in younger subjects ( $\leq 40$ years) [36]. SDmediated inflammation may be associated with mild to moderate multi-organ damage in mice, including liver, heart, kidney and pancreatic injuries [37]. Evidence has shown that 2 days of SD after cecal ligation and puncture-induced sepsis could increase mortality in both young and old mice [38]. However, the roles and mechanisms of short term SD in systemic inflammation and multi-organ injuries remain unclear. In our present study, we found that the subdiaphragmatic vagus nerve played essential roles in post-septic SD-induced aggravation of systemic inflammation and multi-organ injuries.

Through analyzing fecal microbiota diversity and composition of mice from different experimental groups using 16S rRNA gene sequencing, we found that the relative abundance of Proteobacteria and its subgroups were increased after post-septic SD. It is shown that increased abundance of proteobacteria community in the gut might contribute to low-fat/high-sugar diet-induced liver damage and high-fat dietinduced psychiatric disorders in mice $[39,40]$. Proteobacteria is the dominant phyla in septic patients [41, 42]. To determine the causal link between post-septic SD-induced aggravation of systemic inflammation and gut microbiome, pseudo germ-free mice were constructed as recipients receiving fecal microbiota from septic mice with or without SD. As we expected, pseudo germ-free mice transplanted with microbiome from septic mice with SD exhibited increased systemic inflammation as their donors did. Collectively, our results suggest that gut microbiota composition alteration elicited by SD after sepsis plays key roles in the aggravation of systemic inflammation. It is evidenced that the communication between the brain and the endogenous and exogenous microorganisms in the gut could be modulated by the subdiaphragmatic vagus nerve [19, 43-47]. Our data showed that SDV abrogated post-septic SDmediated aggravation of systemic inflammation and multi-organ injuries. Furthermore, SDV could also reverse the increase in systemic inflammation in pseudo germ-free mice transplanted with microbiome from septic mice with SD, indicating an essential role of gut microbiota-subdiaphragmatic vagus nerve axis in post-septic SD-induced aggravation of systemic inflammation. 
Previous studies demonstrate that the ingestion of beneficial bacteria or selective serotonin reuptake inhibitors could alleviate stress-induced anxiety and depression-like behaviors via the subdiaphragmatic vagus nerve, which could be abolished by SDV $[47,48]$. We previously found that LPS at a dose of 0.5 $\mathrm{mg} / \mathrm{kg}$ produces a depression-like phenotype and triggers inflammation via the subdiaphragmatic vagus nerve [19]. Abnormal composition of gut microbiota is observed after administration of $0.5 \mathrm{mg} / \mathrm{kg} \mathrm{LPS}$. Combined with our present data, we speculate that the dominant specific microorganisms present in the intestinal flora under different pathological or experimental circumstances may affect systemic inflammatory response through the subdiaphragmatic vagus nerve system, although further study is needed to confirm this hypothesis. In our present study, the increased abundance of proteobacteria community in the gut after post-septic SD may aggravate systemic inflammation and multi-organ injuries via the subdiaphragmatic vagus nerve.

Studies have shown that splenectomy strongly affected the acute immuno-inflammatory response to polytrauma and secondary sepsis by dampening antigen presentation capacity, specific pro-inflammatory mediators release and granulocyte activation [49]. Significant protective impact of splenectomy has also been shown in stroke [50] and bacterial translocation after burn trauma [51]. We previously found that splenectomy prior to chronic and repeated short-term sleep restrictioncould abrogate the enhancement of LPS-induced increases in systemic inflammation and neuroinflammation [23]. It is shown that pleen development is modulated by neonatal gut microbiota [52], and spleen is essential for gut microbiota disorder-induced immune dysfunction after sleep restriction [22]. In our present study, we found that spleen was essential to the alteration of gut microbiota composition-induced aggravation of systemic inflammation after septic SD. A recent study shows that subdiaphragmatic vagus nerve plays an important role in the communication of gut microbiota and spleen [22]. However, whether subdiaphragmatic vagus nerve could mediate the transmission of signals from gut microbiota to the spleen and thus contribute to post-septic SD-induced aggravation of systemic inflammation remains unclear and needs further study.

\section{Conclusion}

Our findings suggest that the increased systemic inflammation induced by SD after sepsis is mediated via the subdiaphragmatic vagus nerve and spleen. The gut microbiota-subdiaphragmatic vagus nerve axis and gut microbiota-spleen axis plays essential roles in post-septic SD-induced aggravation of systemic inflammation and multi-organ injuries. Our findings highlight the detrimental effects of short term sleep disorder after sepsis on the inflammatory organ damage.

\section{Declarations}

\section{Ethics approval}

Animal protocols were performed in accordance with the National Institute of Health $(\mathrm{NIH})$ Guide for the Care and Use of Laboratory Animals (publications no.80-23) revised 1996 and approved by the 
committee of experimental animals of Tongji Medical College (Wuhan, China).

Consent for publication

Not applicable.

\section{Availability of data and materials}

The datasets generated and/or analyzed during the current study are available in the laboratory of Wuhan union hospital, and the datasets are available from the corresponding author on request.

\section{Competing interests}

The authors declare that they have no competing interests.

\section{Funding}

This work was supported by a grant from the China International Medical Foundation: Special Research Fund for Middle-aged and Youth (Z-2018-35-1902).

\section{Authors' contributions}

Conceived and designed the experiments: SyY and JcZ. Performed the experiments: JcZ, YjZ, BX, ZL, and HQ. Analyzed the data: SyY, JcZ and YjZ. Wrote the paper: YyZ. Revised and edited the manuscript: SyY and JcZ.

\section{Acknowledgements}

The authors thank Hua Yao for technical assistance.

\section{References}

1. Hotchkiss RS, Karl IE: The pathophysiology and treatment of sepsis. N Engl J Med 2003, 348(2):138150.

2. Cavaillon JM, Adib-Conquy M: Monocytes/macrophages and sepsis. CRIT CARE MED 2005, 33(12 Suppl):S506-S509.

3. Carlson DE, Chiu WC: The absence of circadian cues during recovery from sepsis modifies pituitaryadrenocortical function and impairs survival. SHOCK 2008, 29(1):127-132.

4. Pulak LM, Jensen L: Sleep in the Intensive Care Unit: A Review. J INTENSIVE CARE MED 2016, 31(1):14-23.

5. Pisani MA, Friese RS, Gehlbach BK, Schwab RJ, Weinhouse GL, Jones SF: Sleep in the intensive care unit. Am J Respir Crit Care Med 2015, 191(7):731-738. 
6. Beltrami FG, Nguyen XL, Pichereau C, Maury E, Fleury B, Fagondes S: Sleep in the intensive care unit. J BRAS PNEUMOL 2015, 41(6):539-546.

7. Besedovsky L, Lange T, Born J: Sleep and immune function. Pflugers Arch 2012, 463(1):121-137.

8. Kamdar BB, Needham DM, Collop NA: Sleep deprivation in critical illness: its role in physical and psychological recovery. J INTENSIVE CARE MED 2012, 27(2):97-111.

9. Hardin KA: Sleep in the ICU: potential mechanisms and clinical implications. CHEST 2009, 136(1):284-294.

10. Parthasarathy S, Tobin MJ: Sleep in the intensive care unit. Intensive Care Med 2004, 30(2):197-206.

11. Benedict C, Vogel H, Jonas W, Woting A, Blaut M, Schurmann A, Cedernaes J: Gut microbiota and glucometabolic alterations in response to recurrent partial sleep deprivation in normal-weight young individuals. MOL METAB 2016, 5(12):1175-1186.

12. Poroyko VA, Carreras A, Khalyfa A, Khalyfa AA, Leone V, Peris E, Almendros I, Gileles-Hillel A, Qiao Z, Hubert N et al: Chronic Sleep Disruption Alters Gut Microbiota, Induces Systemic and Adipose Tissue Inflammation and Insulin Resistance in Mice. Sci Rep 2016, 6:35405.

13. Gill SR, Pop M, Deboy RT, Eckburg PB, Turnbaugh PJ, Samuel BS, Gordon JI, Relman DA, FraserLiggett CM, Nelson KE: Metagenomic analysis of the human distal gut microbiome. SCIENCE 2006, 312(5778):1355-1359.

14. Borovikova LV, Ivanova S, Zhang M, Yang H, Botchkina GI, Watkins LR, Wang H, Abumrad N, Eaton $\mathrm{JW}$, Tracey KJ: Vagus nerve stimulation attenuates the systemic inflammatory response to endotoxin. NATURE 2000, 405(6785):458-462.

15. Bernik TR, Friedman SG, Ochani M, DiRaimo R, Ulloa L, Yang H, Sudan S, Czura CJ, Ivanova SM, Tracey KJ: Pharmacological stimulation of the cholinergic antiinflammatory pathway. J EXP MED 2002, 195(6):781-788.

16. Wang $\mathrm{H}$, Yu M, Ochani M, Amella CA, Tanovic M, Susarla S, Li JH, Wang H, Yang H, Ulloa L et al: Nicotinic acetylcholine receptor alpha7 subunit is an essential regulator of inflammation. NATURE 2003, 421(6921):384-388.

17. Bernik TR, Friedman SG, Ochani M, DiRaimo R, Susarla S, Czura CJ, Tracey KJ: Cholinergic antiinflammatory pathway inhibition of tumor necrosis factor during ischemia reperfusion. $J$ VASC SURG 2002, 36(6):1231-1236.

18. Guarini S, Altavilla D, Cainazzo MM, Giuliani D, Bigiani A, Marini H, Squadrito G, Minutoli L, Bertolini $\mathrm{A}$, Marini $\mathrm{R}$ et al: Efferent vagal fibre stimulation blunts nuclear factor-kappaB activation and protects against hypovolemic hemorrhagic shock. CIRCULATION2003, 107(8):1189-1194.

19. Zhang J, Ma L, Chang L, Pu Y, Qu Y, Hashimoto $K$ : A key role of the subdiaphragmatic vagus nerve in the depression-like phenotype and abnormal composition of gut microbiota in mice after lipopolysaccharide administration. Trans/ Psychiatry 2020, 10(1):186.

20. Lewis SM, Williams A, Eisenbarth SC: Structure and function of the immune system in the spleen. Sci Immunol 2019, 4(33). 
21. Rosado MM, Aranburu A, Scarsella M, Cascioli S, Giorda E, Del CF, Mortera SL, Mortari EP, Petrini S, Putignani $L$ et al: Spleen development is modulated by neonatal gut microbiota. IMMUNOL LETT 2018, 199:1-15.

22. Wang G, Wu X, Zhu G, Han S, Zhang J: Dexmedetomidine alleviates sleep-restriction-mediated exaggeration of postoperative immunosuppression via splenic TFF2 in aged mice. Aging (Albany NY) 2020, 12(6):5318-5335.

23. Xu D, Zhang YJ, Xie B, Yao H, Yuan Y, Yuan SY, Zhang JC: The spleen mediates chronic sleep restriction-mediated enhancement of LPS-induced neuroinflammation, cognitive deficits, and anxietylike behavior. Aging 2020.

24. Nunes J, Apostolico JS, Andrade D, Ruiz FS, Fernandes ER, Andersen ML, Keller AC, Rosa DS: Sleep deprivation predisposes allergic mice to neutrophilic lung inflammation. J Allergy Clin Immuno/ 2018, 141(3):1018-1027.

25. Gao T, Wang Z, Dong Y, Cao J, Lin R, Wang X, Yu Z, Chen Y: Role of melatonin in sleep deprivationinduced intestinal barrier dysfunction in mice. J PINEAL RES 2019, 67(1):e12574.

26. Zhan G, Yang N, Li S, Huang N, Fang X, Zhang J, Zhu B, Yang L, Yang C, Luo A: Abnormal gut microbiota composition contributes to cognitive dysfunction in SAMP8 mice. Aging (Albany NY) 2018, 10(6):1257-1267.

27. Bermon S, Petriz B, Kajeniene A, Prestes J, Castell L, Franco OL: The microbiota: an exercise immunology perspective. EXERC IMMUNOL REV2015, 21:70-79.

28. Simpson N, Dinges DF: Sleep and inflammation. NUTR REV2007, 65(12 Pt 2):S244-S252.

29. Irwin MR, Wang M, Campomayor CO, Collado-Hidalgo A, Cole S: Sleep deprivation and activation of morning levels of cellular and genomic markers of inflammation. Arch Intern Med 2006, 166(16):1756-1762.

30. Irwin MR, Wang M, Ribeiro D, Cho HJ, Olmstead R, Breen EC, Martinez-Maza O, Cole S: Sleep loss activates cellular inflammatory signaling. Biol Psychiatry 2008, 64(6):538-540.

31. Mullington JM, Simpson NS, Meier-Ewert HK, Haack M: Sleep loss and inflammation. Best Pract Res Clin Endocrinol Metab 2010, 24(5):775-784.

32. Wright KJ, Drake AL, Frey DJ, Fleshner M, Desouza CA, Gronfier C, Czeisler CA: Influence of sleep deprivation and circadian misalignment on cortisol, inflammatory markers, and cytokine balance. BRAIN BEHAV IMMUN 2015, 47:24-34.

33. Haack M, Sanchez E, Mullington JM: Elevated inflammatory markers in response to prolonged sleep restriction are associated with increased pain experience in healthy volunteers. SLEEP 2007, 30(9):1145-1152.

34. Irwin MR, Olmstead R, Carroll JE: Sleep Disturbance, Sleep Duration, and Inflammation: A Systematic Review and Meta-Analysis of Cohort Studies and Experimental Sleep Deprivation. Biol Psychiatry 2016, 80(1):40-52.

35. Kroller-Schon S, Daiber A, Steven S, Oelze M, Frenis K, Kalinovic S, Heimann A, Schmidt FP, Pinto A, Kvandova $\mathrm{M}$ et al: Crucial role for Nox2 and sleep deprivation in aircraft noise-induced vascular and 
cerebral oxidative stress, inflammation, and gene regulation. EUR HEART J 2018, 39(38):3528-3539.

36. Carroll JE, Carrillo C, Olmstead R, Witarama T, Breen EC, Yokomizo M, Seeman T, Irwin MR: Sleep deprivation and divergent toll-like receptor-4 activation of cellular inflammation in aging. SLEEP 2015, 38(2):205-211.

37. Periasamy S, Hsu DZ, Fu YH, Liu MY: Sleep deprivation-induced multi-organ injury: role of oxidative stress and inflammation. EXCLI J 2015, 14:672-683.

38. Friese RS, Bruns B, Sinton CM: Sleep deprivation after septic insult increases mortality independent of age. J Trauma 2009, 66(1):50-54.

39. Sen T, Cawthon CR, Ihde BT, Hajnal A, DiLorenzo PM, de La Serre CB, Czaja K: Diet-driven microbiota dysbiosis is associated with vagal remodeling and obesity. PHYSIOL BEHAV 2017, 173:305-317.

40. Jeong MY, Jang HM, Kim DH: High-fat diet causes psychiatric disorders in mice by increasing Proteobacteria population. NEUROSCI LETT 2019, 698:51-57.

41. Moron R, Galvez J, Colmenero M, Anderson P, Cabeza J, Rodriguez-Cabezas ME: The Importance of the Microbiome in Critically III Patients: Role of Nutrition. NUTRIENTS 2019, 11(12).

42. Wan YD, Zhu RX, Wu ZQ, Lyu SY, Zhao LX, Du ZJ, Pan XT: Gut Microbiota Disruption in Septic Shock Patients: A Pilot Study. Med Sci Monit 2018, 24:8639-8646.

43. Forsythe $P$, Bienenstock J, Kunze WA: Vagal pathways for microbiome-brain-gut axis communication. ADV EXP MED BIOL 2014, 817:115-133.

44. Bonaz B, Bazin T, Pellissier S: The Vagus Nerve at the Interface of the Microbiota-Gut-Brain Axis. Front Neurosci 2018, 12:49.

45. Cawthon CR, de La Serre CB: Gut bacteria interaction with vagal afferents. BRAIN RES2018, 1693(Pt B):134-139.

46. Bharwani A, West C, Champagne-Jorgensen K, McVey NK, Ruberto J, Kunze WA, Bienenstock J, Forsythe $\mathrm{P}$ : The vagus nerve is necessary for the rapid and widespread neuronal activation in the brain following oral administration of psychoactive bacteria. NEUROPHARMACOLOGY 2020, 170:108067.

47. Bravo JA, Forsythe P, Chew MV, Escaravage E, Savignac HM, Dinan TG, Bienenstock J, Cryan JF: Ingestion of Lactobacillus strain regulates emotional behavior and central GABA receptor expression in a mouse via the vagus nerve. Proc Nat/ Acad Sci U S A 2011, 108(38):16050-16055.

48. McVey NK, Bienenstock J, Bharwani A, Champagne-Jorgensen K, Mao Y, West C, Liu Y, Surette MG, Kunze W, Forsythe $\mathrm{P}$ : Oral selective serotonin reuptake inhibitors activate vagus nerve dependent gutbrain signalling. Sci Rep 2019, 9(1):14290.

49. Drechsler S, Zipperle J, Rademann P, Jafarmadar M, Klotz A, Bahrami S, Osuchowski MF: Splenectomy modulates early immuno-inflammatory responses to trauma-hemorrhage and protects mice against secondary sepsis. Sci Rep 2018, 8(1):14890.

50. Chauhan A, Al MA, Spiegel G, Harris N, Zhu L, McCullough LD: Splenectomy protects aged mice from injury after experimental stroke. NEUROBIOL AGING 2018, 61:102-111. 
51. Baykal A, Aydin C, Hascelik G, Ayhan A, Korkmaz A, Sayek I: Experimental study of the effects of splenectomy and partial splenectomy on bacterial translocation. J Trauma 1999, 46(6):1096-1099.

52. Rosado MM, Aranburu A, Scarsella M, Cascioli S, Giorda E, Del CF, Mortera SL, Mortari EP, Petrini S, Putignani $L$ et al: Spleen development is modulated by neonatal gut microbiota. IMMUNOL LETT 2018, 199:1-15.

Figures 
a

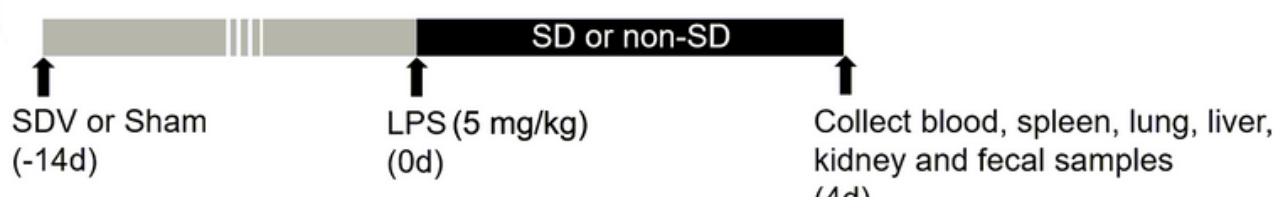

$(-14 d)$

(Od) kidney and fecal samples

b

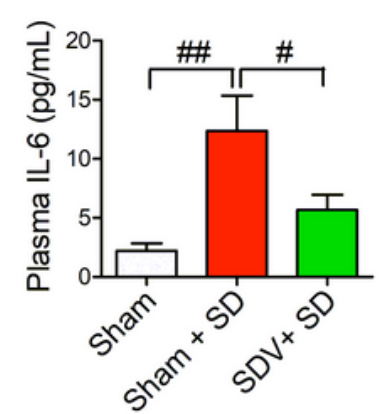

e

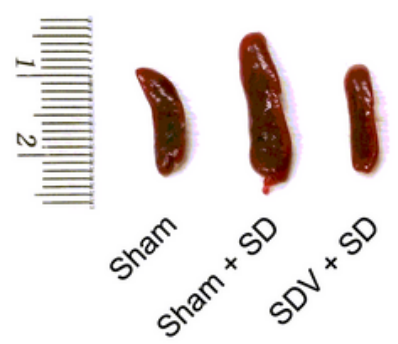

f

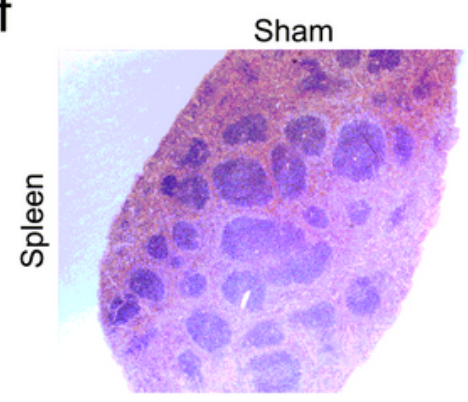

g

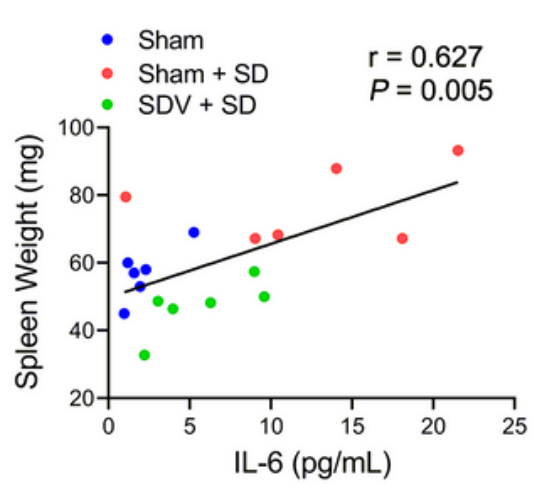

C
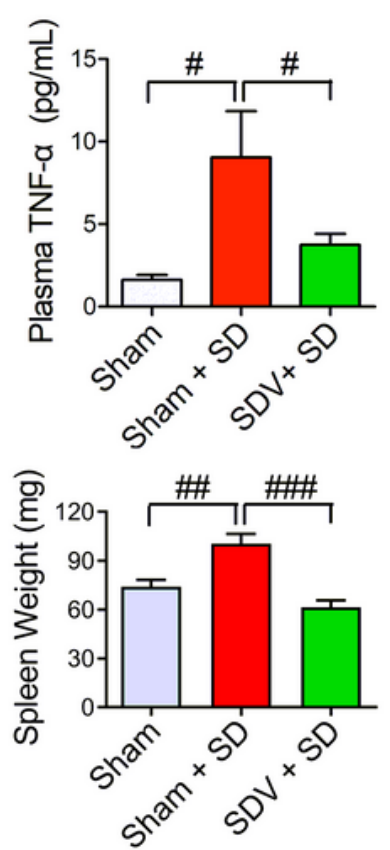

Sham + SD

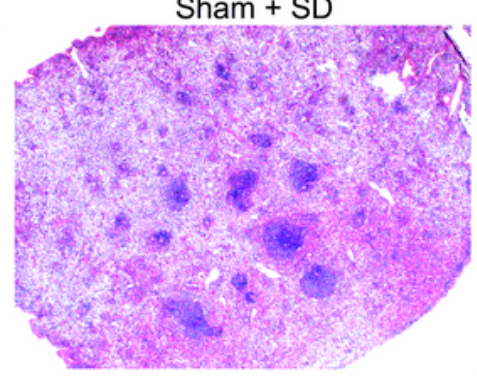

h

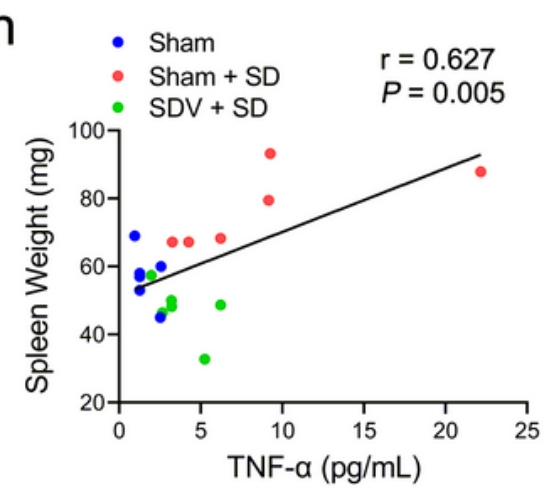

d
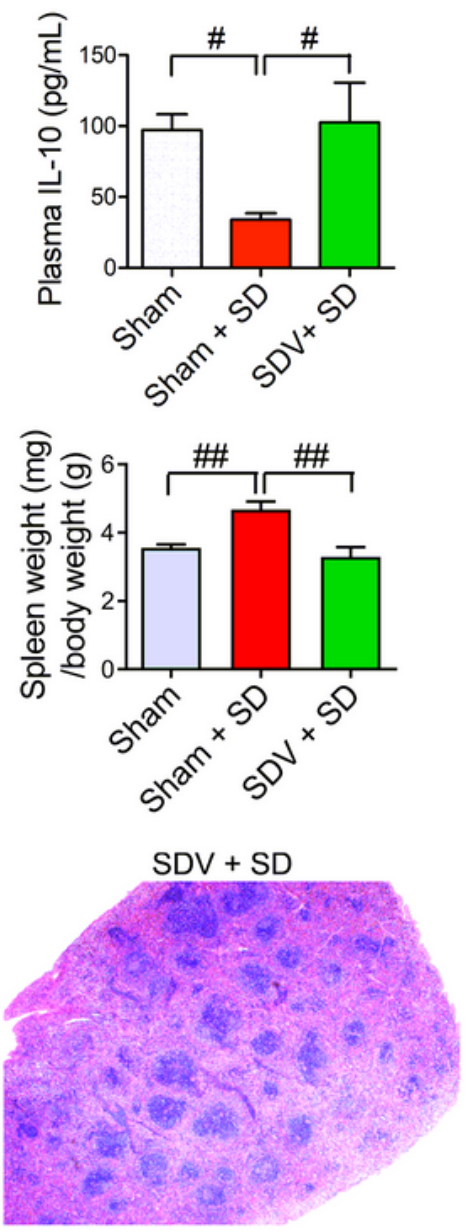

\section{Figure 1}

Effects of subdiaphragmatic vagotomy on systemic inflammation in septic mice with or without sleep deprivation. (a) Treatment schedule. Mice were administered with lipopolysaccharide (LPS) at a dose of 5 $\mathrm{mg} / \mathrm{kg}$, followed by 3 days of sleep deprivation. Subdiaphragmatic vagotomy (SDV) or sham operation was performed 14 days prior to LPS administration. On day 4 after LPS administration, the blood, spleen, lung, liver, kidney and fecal samples were collected. $(b-d)$ The plasma levels of IL-6, TNF- $a$ and IL-10 in 
each group were measured by enzyme linked immunosorbent assays (ELISA). SDV abrogated post-septic sleep deprivation (SD)-mediated increase in the plasma levels of IL- 6 and TNF-a. The decrease in the plasma level of IL-10 after post-septic SD was also abrogated by SDV. (e) Spleen weight and spleen/body weight ratio in each group. SDV abrogated post-septic SD-mediated increase in the spleen weight and spleen/body weight ratio. (f) The pathological changes in the spleen were observed using spleen sections stained with hematoxylin-eosin by light microscopy $(\times 200)$. (G) Correlation analysis of plasma IL-6 level with spleen weight $(r=0.005, P=0.627)$. Correlation analysis of spleen weight with IL-6 plsama level $(g)$ or TNF-a plsama level (h). There was a positive correlation between spleen weight with IL-6 plsama level $(r=0.604, P=0.005)$ or TNF-a plsama level $(r=0.627, P=0.008)$. Data are shown as mean \pm SEM $(n=$ 6/group). \#P $<0.05, \# \# P<0.01$, \#\#\#P $<0.001$.
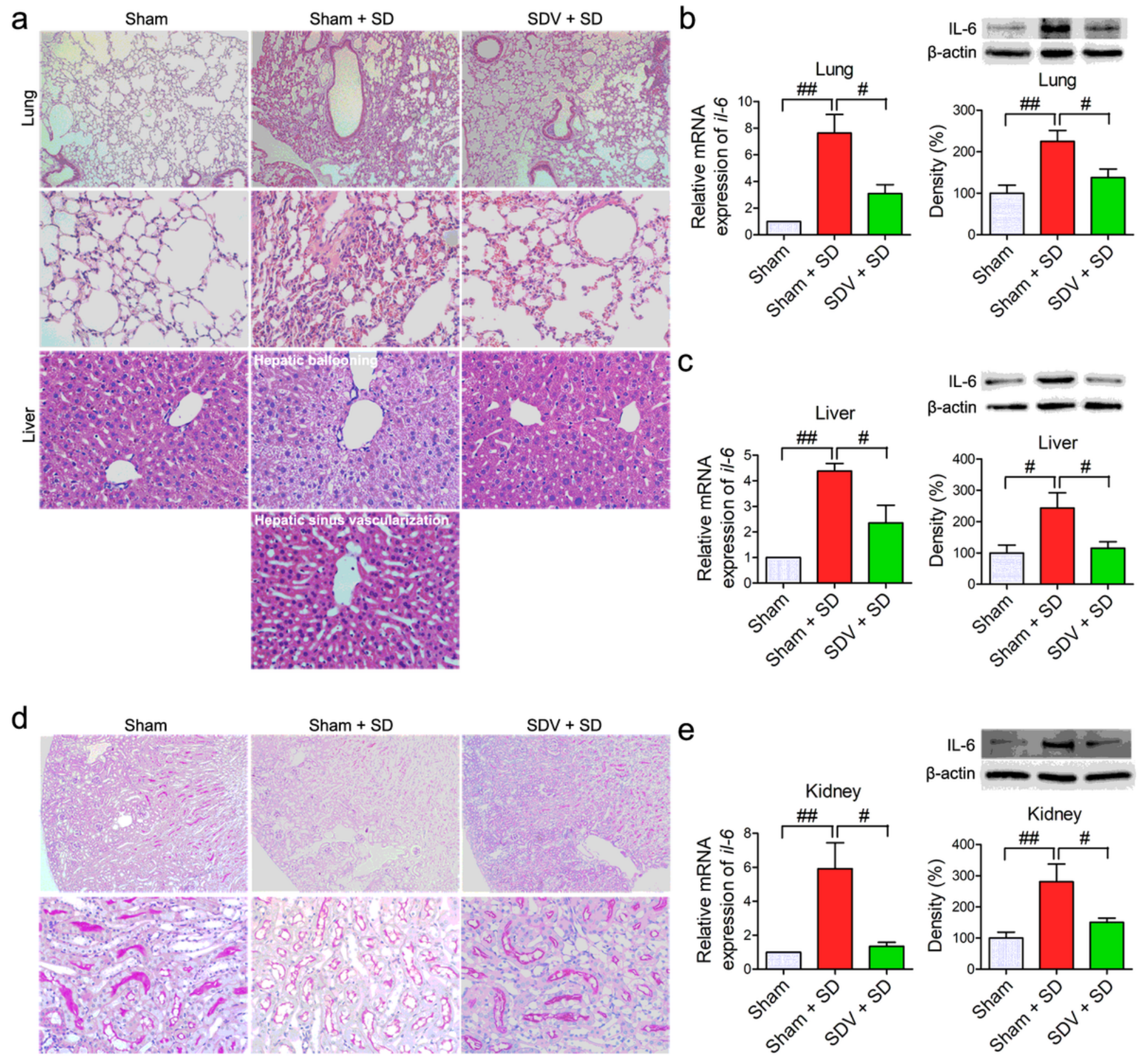
Effects of subdiaphragmatic vagotomy on organ injuries in septic mice with or without sleep deprivation.

(a) The pathological changes in the lung and liver were observed using lung and liver sections stained with hematoxylin-eosin by light microscopy $(\times 100$ or $\times 200)$. (b, c) The mRNA and protein expression of IL6 in the lung and liver were measured by quantitative Real-Time PCR (RT-qPCR) and western blotting. Subdiaphragmatic vagotomy (SDV) abrogated post-septic sleep deprivation (SD)-mediated increase in the expression of IL-6 in the lung and liver. (d) The pathological changes in the kidney were observed using kidney sections stained with periodic acid-schiff (PAS) by light microscopy ( $\times 100$ or $\times 200)$. (e) RT-qPCR and western blotting analysis of the mRNA and protein expression of IL- 6 in the kidney. SDV abrogated post-septic SD-mediated increase in IL-6 expression in the kidney. Data are shown as mean \pm SEM ( $n=6 /$ group). \#P $<0.05$, \#\#P $<0.01$.

a

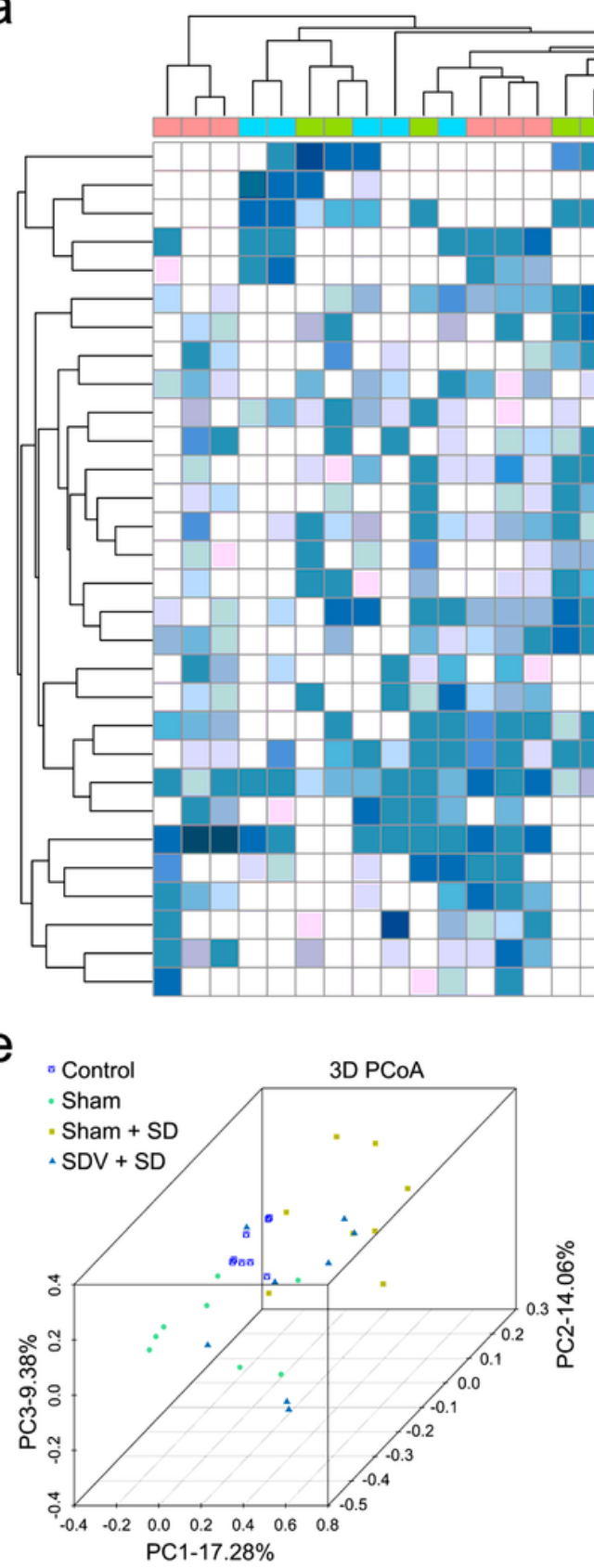

b

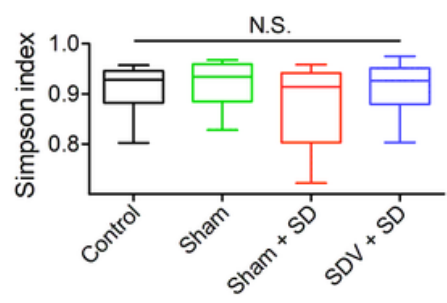

C

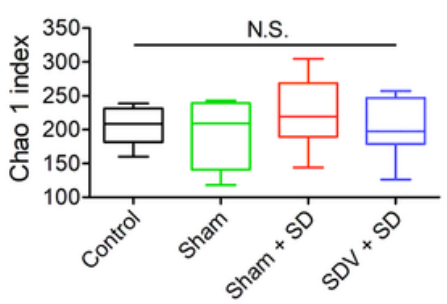

d

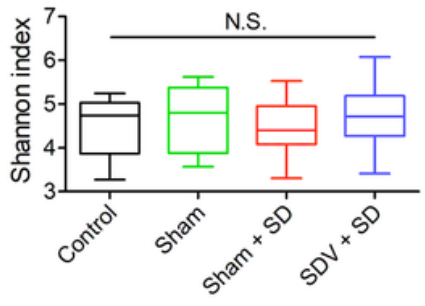

- Control

= Sham

- Sham + SD

SDV + SD 


\section{Figure 3}

Effects of subdiaphragmatic vagotomy on fecal microbiota diversity in septic mice with sleep deprivation. (a) Heat map of different abundance of fecal bacteria between the groups. There were no significant differences in the richness of intestinal flora determined via Shannon index (b), Chao 1 (c), Simpson index (d), 3D-Principal Coordinate Analysis (3D-PCoA; e) and Principal Component Analysis (PCA; f). Data are shown as mean \pm SEM ( $n=8$ /group). N.S., not significant.

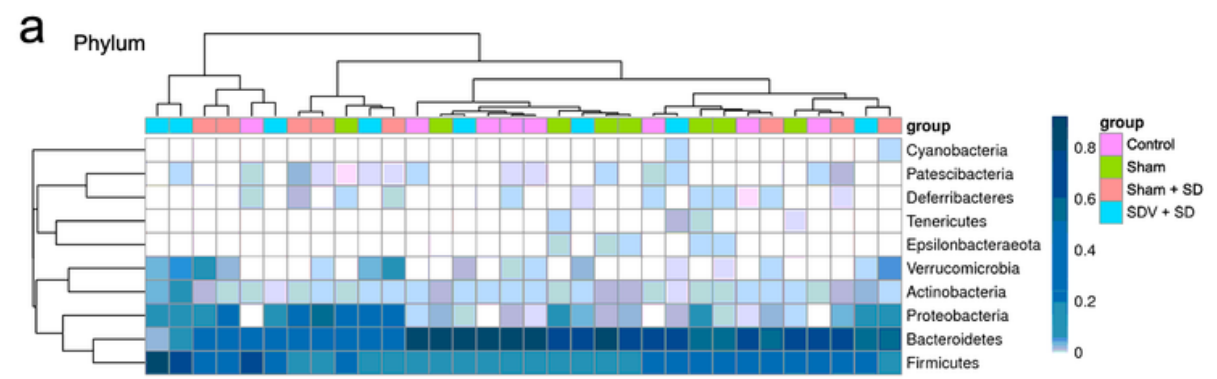

b
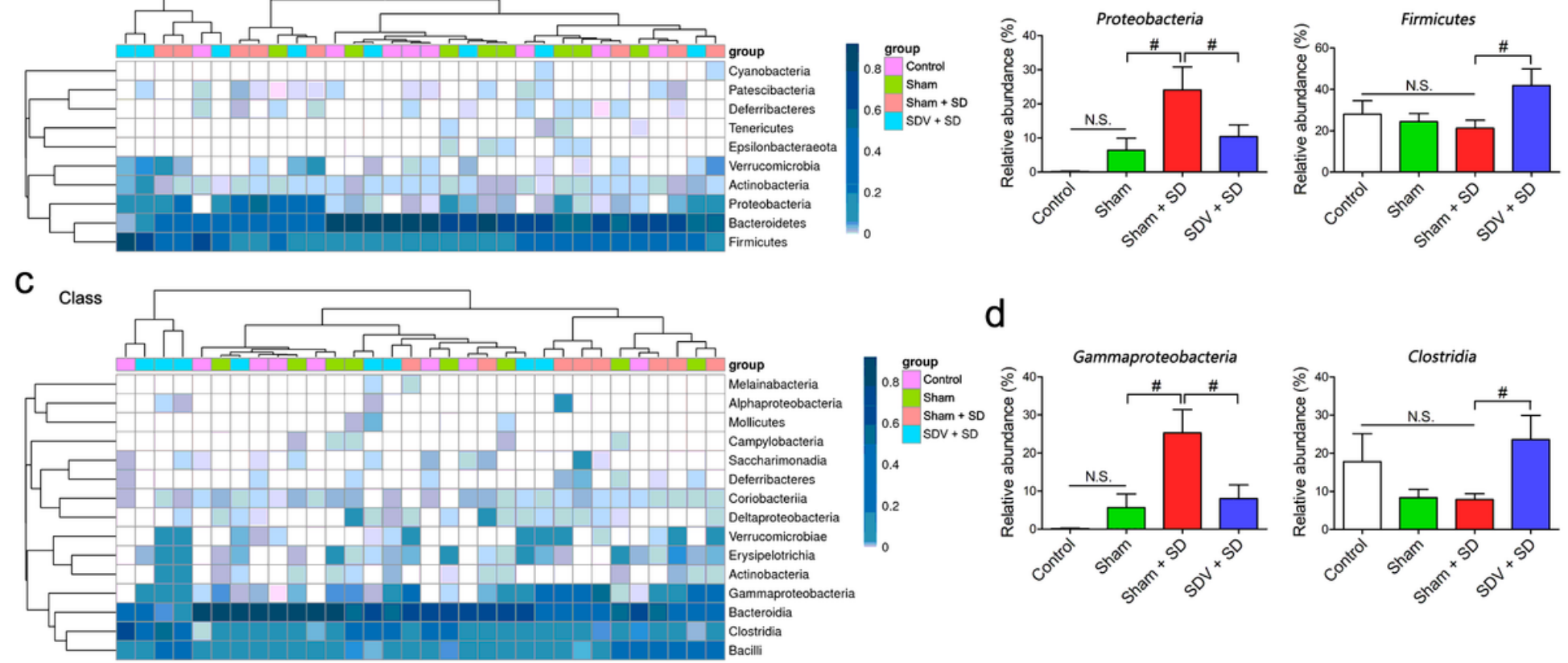

d
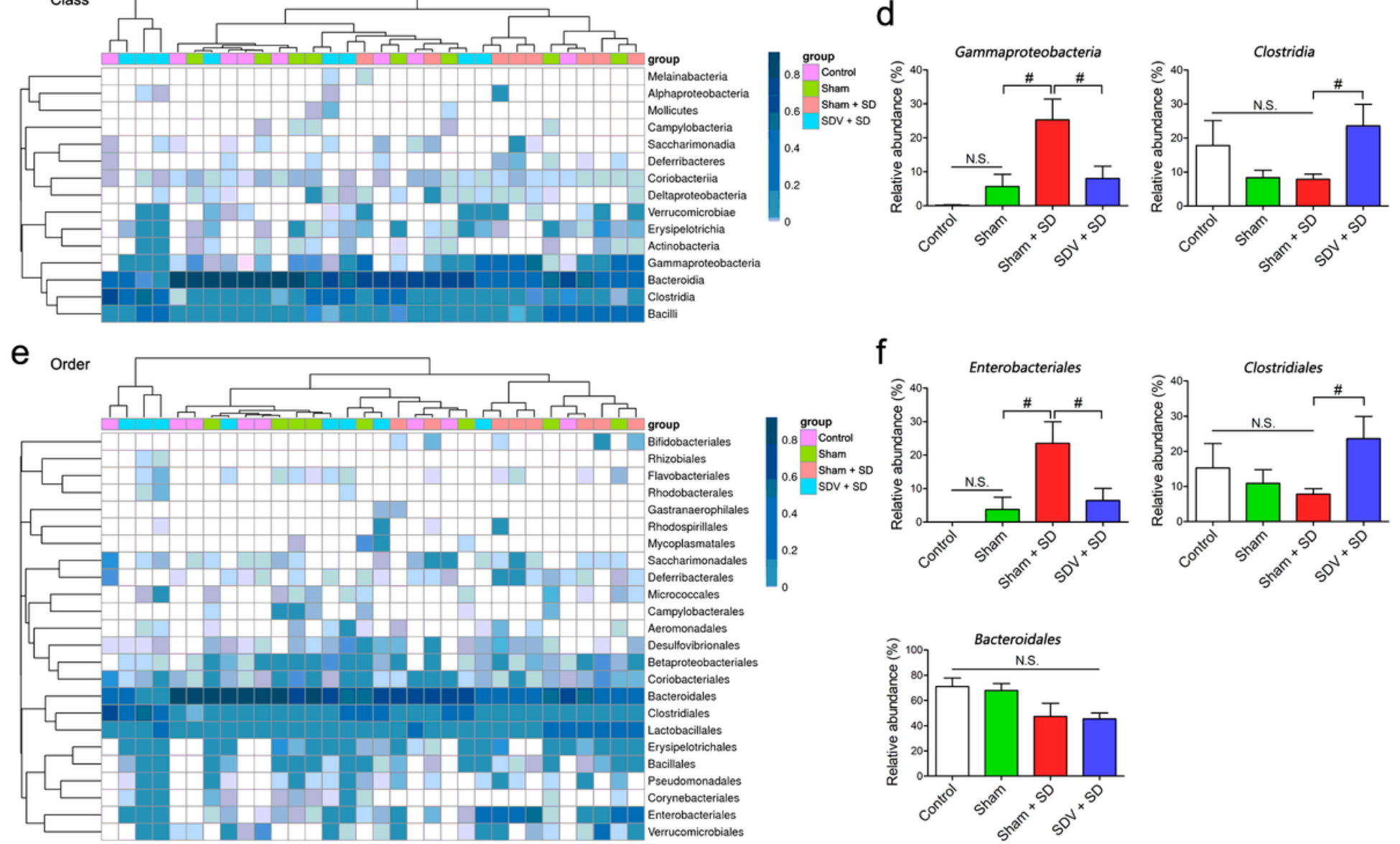

\section{Figure 4}

Effects of subdiaphragmatic vagotomy on fecal microbiota composition at phylum, class and order levels. $(a, b)$ Relative abundance at the phylum level between the groups. Post-septic sleep deprivation (SD) significantly increased the relative abundance of Proteobacteria, which was abrogated by subdiaphragmatic vagotomy (SDV). (c, d) Relative abundance at the class level between the groups. SDV 
abrogated post-septic SD-induced increase in the relative abundance of Gammaproteobacteria. $(e, f)$ Relative abundance at the order level between the groups. SDV abrogated post-septic SD-induced increase in the relative abundance of Enterobacteriales. Data are shown as mean \pm SEM ( $n=8 /$ group). N.S., not significant. \#P $<0.05$.
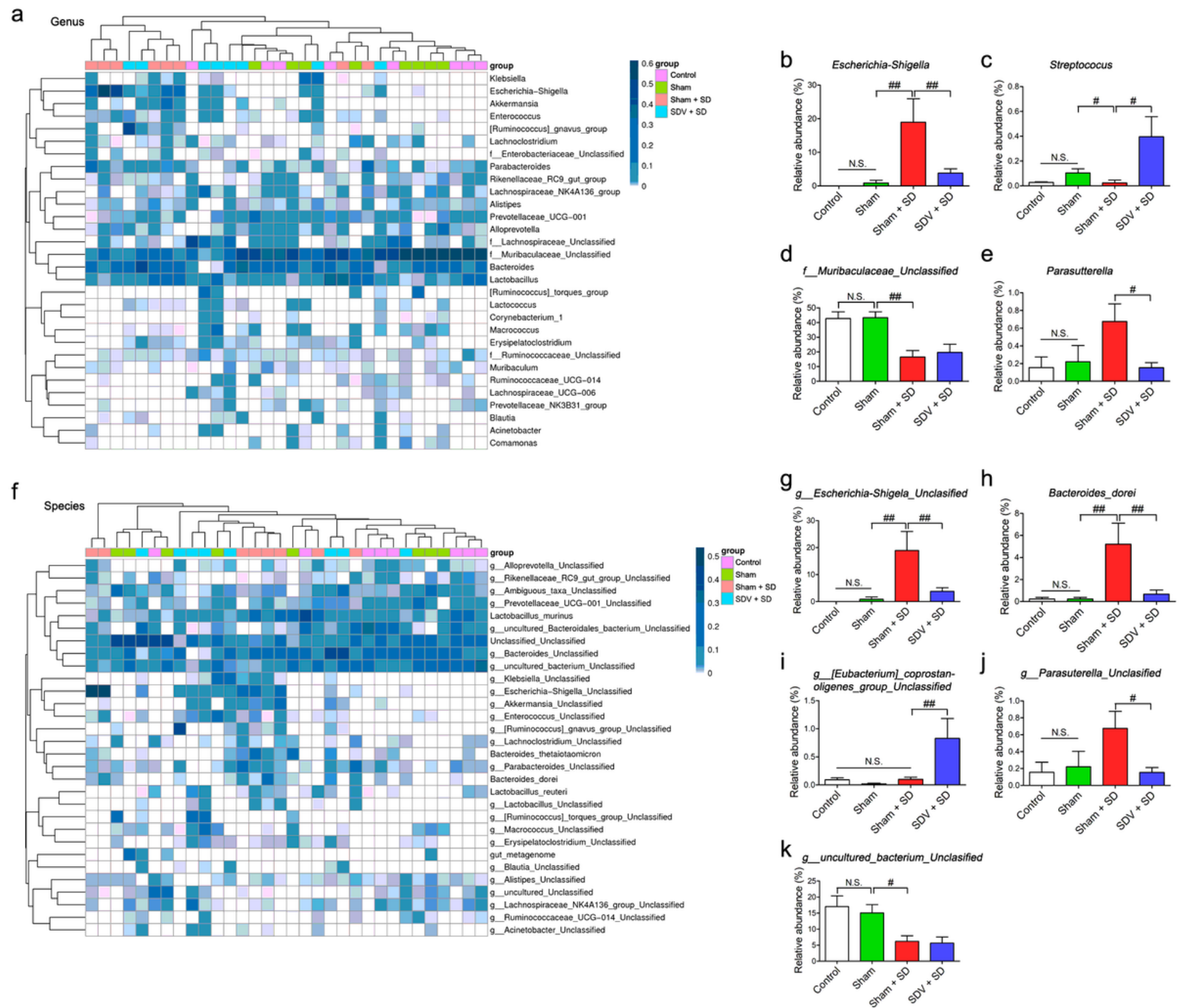

\section{Figure 5}

Effects of subdiaphragmatic vagotomy on fecal microbiota composition at the levels of genus and species. $(a-e)$ Relative abundance at the genus level between the groups. Post-septic sleep deprivation (SD) significantly increased the relative abundance of Escherichia Shigella, which was abrogated by subdiaphragmatic vagotomy (SDV). SDV abrogated post-septic SD-induced decrease in the relative abundance of Streptococus. $(f-k)$ Relative abundance at the species level between the groups. SDV abrogated post-septic SD-induced increase in the relative abundance of g_Escherichia- 
Shigela_Unclassified and Bacteroides_dorei. Data are shown as mean \pm SEM ( $\mathrm{n}=8 /$ group). N.S., not significant. \#P $<0.05$ and \#\#P $<0.01$.
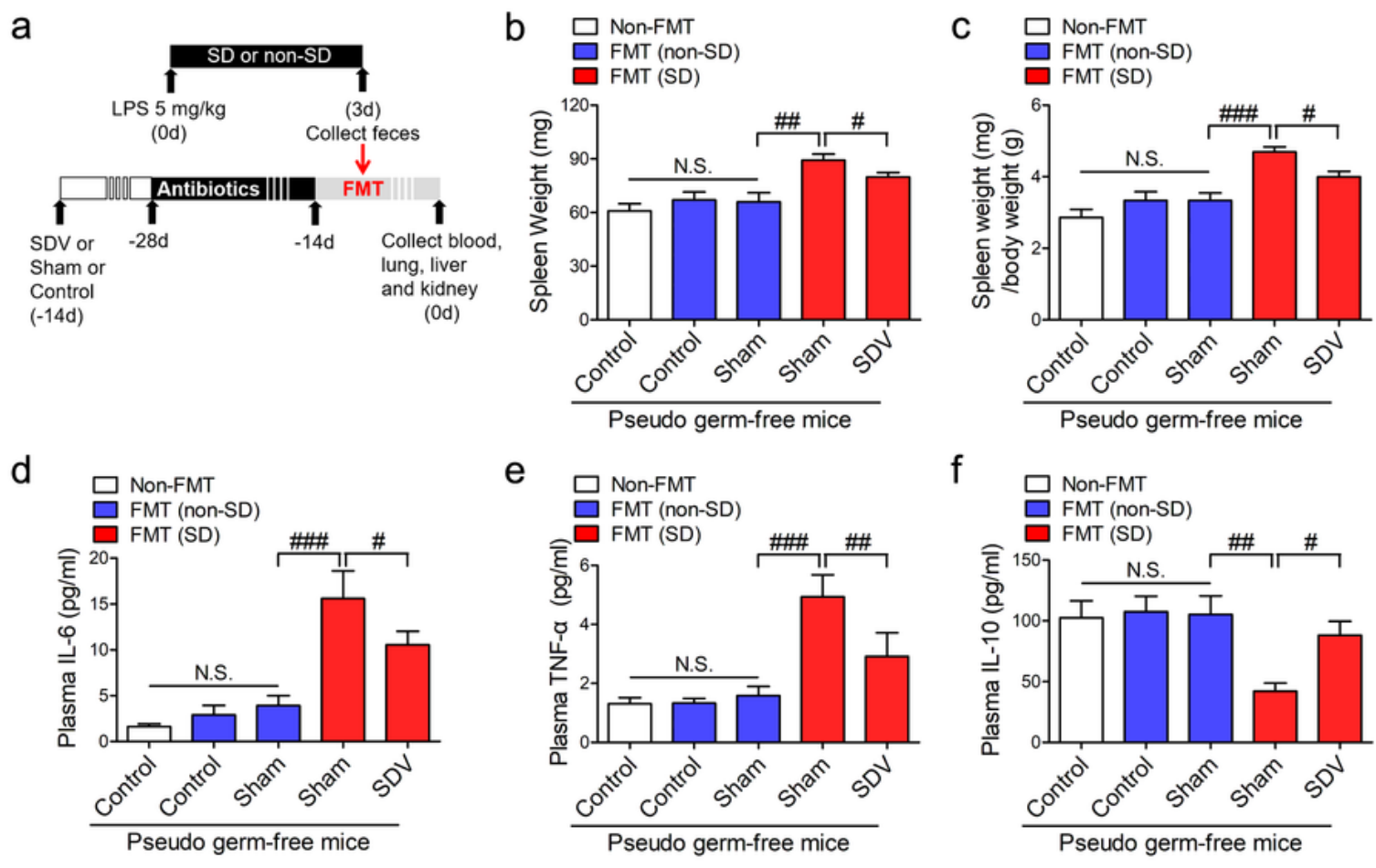

e
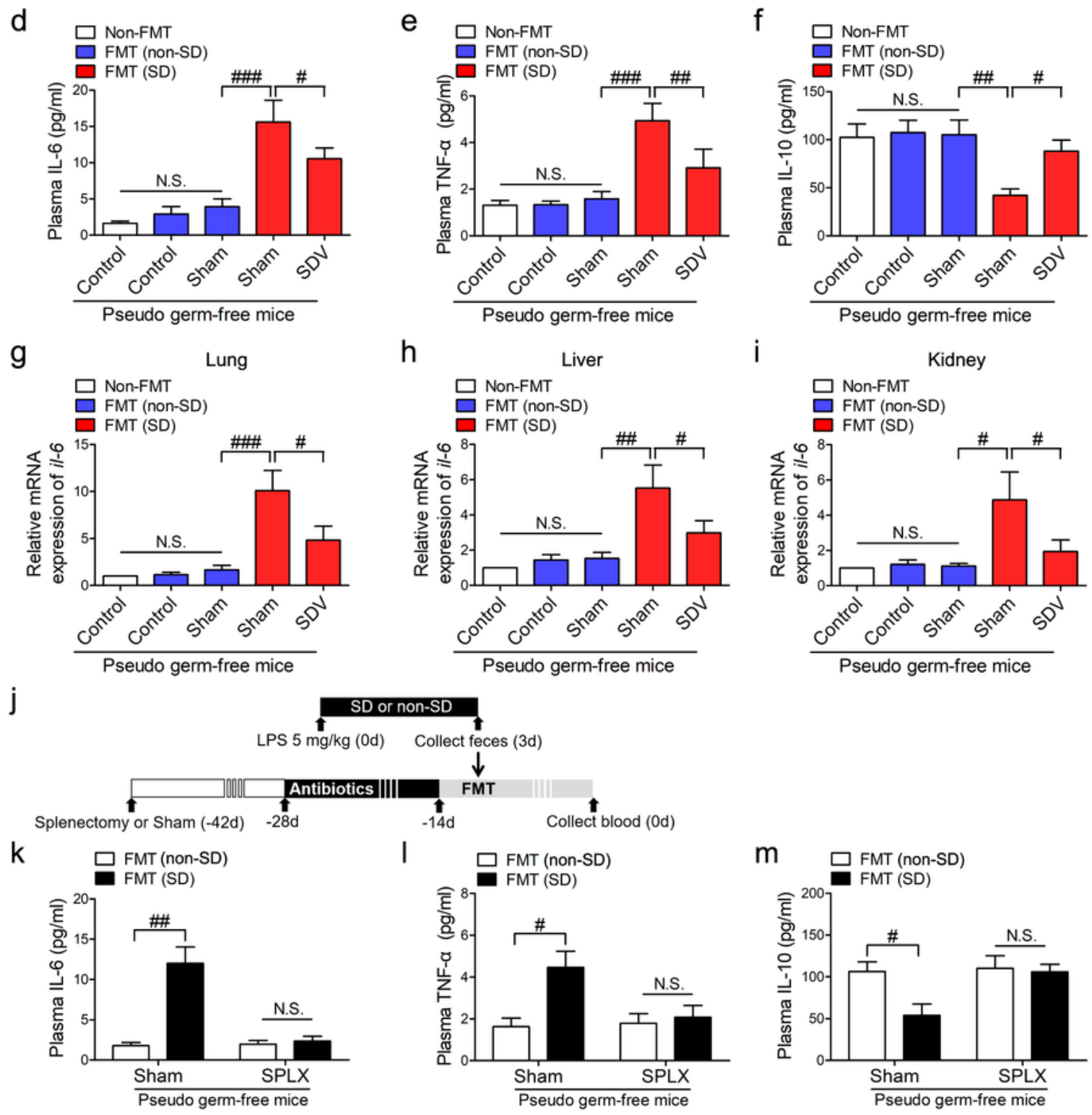

Figure 6

Effects of gut microbiota-vagus nerve axis and gut microbiota-spleen axis on systemic inflammation. (a) Treatment schedule. The pseudo germ-free mouse model was created by administering antibiotics for 14 consecutive days, and then fecal microbiota transplant (FMT) was performed by gavaging supernatant 
from fecal suspension of septic mice with or without sleep deprivation (SD) into pseudo germ-free mice with or without subdiaphragmatic vagotomy (SDV). Spleen weight (b) and spleen/body weight ratio (c) in each group. The spleen weight and spleen/body weight ratio were significantly increased in pseudo germfree mice transplanted with fecal suspension from septic mice with SD, which was abrogated by SDV. (df) The plasma levels of IL-6, TNF- $a$ and IL-10 in each group were measured by enzyme linked immunosorbent assays (ELISA). SDV abrogated the increase in the plasma levels of IL- 6 and TNF- $a$ and the decrease in the plsama IL-10 level in pseudo germ-free mice transplanted with fecal suspension from septic mice with SD. ( $\mathrm{g}-\mathrm{i}$ ) The mRNA expression of IL- 6 in the lung, liver and kidney were measured by quantitative Real-Time PCR. The mRNA expression of IL- 6 in the lung, liver and kidney was significantly increased in pseudo germ-free mice transplanted with fecal suspension from septic mice with SD, which was abrogated by SDV. (j) Treatment schedule. The pseudo germ-free mice with or without splenectomy were received FMT from septic mice with or without SD. $(k-m)$ The plasma levels of IL-6, TNF-a and IL-10 in each group were measured by ELISA. Splenectomy abrogated the increase in the plsama levels of IL-6 and TNF- $a$ and the decrease in the plsama IL-10 level in pseudo germ-free mice transplanted with fecal suspension from septic mice with SD. Data are shown as mean \pm SEM $(n=6 /$ group $)$. N.S., not significant. $\# \mathrm{P}<0.05, \# \# \mathrm{P}<0.01$, \#\#\#P $<0.001$.

\section{Supplementary Files}

This is a list of supplementary files associated with this preprint. Click to download.

- Graphicalabstract.tif

- Graphicalabstract.tif 